T. NISHIMURA

KODAI MATH. J.

9 (1986), 188-190

\title{
TOPOLOGICAL INVARIANCE OF WEIGHTS FOR WEIGHTED HOMOGENEOUS SINGULARITIES
}

\author{
BY TAKAHASHI NISHIMURA
}

\section{§1. Introduction}

A polynomial $f\left(z_{1}, \cdots, z_{n}\right)$ is called weighted homogeneous with weights $\left(r_{1}, \cdots, r_{n}\right) \in \boldsymbol{Q}^{n}$ if $i_{1} r_{1}+\cdots+i_{n} r_{n}=1$ for any monomial $\alpha z_{1}^{2_{1}} \cdots z_{n}^{2 n}$ of $f$, and non-degenerate if $\left\{\partial f / \partial z_{1}(z)=\cdots=\partial f / \partial z_{n}(z)=0\right\}=\{0\}$ as germs at the origin of $\boldsymbol{C}^{n}$. Then it arises the problem whether the topological type of $\left(\boldsymbol{C}^{n}, f^{-1}(0)\right)$ determines weights of non-degenerate weighted homogeneous polynomial $f$. This problem has been proved affirmatively by Yoshinaga-Suzuki for the case $n=2$, namely,

THEOREM ([7]). Let $f_{i}\left(z_{1}, z_{2}\right)(i=1,2)$ be non-degenerate weighted homogeneous polynomials with weights $\left(r_{i 1}, r_{i 2}\right)$ such that $0<r_{i 1} \leqq r_{i 2} \leqq 1 / 2$. If $\left(\boldsymbol{C}^{2}, f_{1}^{-1}(0)\right)$ is relatively homeomorphic to $\left(\boldsymbol{C}^{2}, f_{2}^{-1}(0)\right)$, then $\left(r_{11}, r_{12}\right)=\left(r_{21}, r_{22}\right)$.

In this paper we give a simple proof of the above theorem. Our method is much more geometric and makes clear the topological structure of non-degenerate weighted homogeneous singularities for the case $n=2$. For the case $n=3$, Orlik [4] proved the above problem affirmatively. Our method is different from the one due to Orlik.

The author wishes to thank Takuo Fukuda, Etsuo Yoshinaga, Masahiko Suzuki, and Hiroshi Noguchi for helpful comments and suggestions.

\section{§. Proof of the theorem}

For any non-degenerate weighted homogeneous polynomial $f$ with weights $\left(r_{1}, r_{2}\right)$ and any point $\boldsymbol{z}=\left(z_{1}, z_{2}\right) \in \boldsymbol{C}^{2}-\{0\}$, we denote the set $\left\{\boldsymbol{w}=\left(w_{1}, w_{2}\right) \mid w_{\text {, }}\right.$ $\left.=\exp \left(2 \pi \sqrt{-1} r_{j} t\right) z_{j}, t \in \boldsymbol{R}\right\}$ by $\boldsymbol{C}_{f}^{*}(\boldsymbol{z})$. Let $a$, and $b$, be relatively prime integers such that $r_{j}=a_{j} / b_{j}$. The following two assertions 1 and 2 are trivial.

ASSERTION 1. The integers $a_{1} \cdot\left[b_{1}, b_{2}\right] / b_{1}$ and $a_{2} \cdot\left[b_{1}, b_{2}\right] / b_{2}$ are relatively prime, where $\left[b_{1}, b_{2}\right]$ denotes the least common multiple for integers $b_{1}, b_{2}$.

ASSERTION 2. For any point $z=\left(z_{1}, z_{2}\right) \in C^{2}-\{0\}$ such that $z_{1} \cdot z_{2} \neq 0$,

Received September 20, 1985 
$\boldsymbol{C}_{f}^{*}(\boldsymbol{z}) \subset|\boldsymbol{z}| S^{3}$ and $\boldsymbol{C}_{f}^{*}(\boldsymbol{z})$ is a torus knot of type $\left(a_{1} \cdot\left[b_{1}, b_{2}\right] / b_{1}, a_{2} \cdot\left[b_{1}, b_{2}\right] / b_{2}\right)$, where $|\boldsymbol{z}| S^{3}$ is the set $\left\{\left.\left(w_{1}, w_{2}\right) \in \boldsymbol{C}^{2}|| w_{1}\right|^{2}+\left|w_{2}\right|^{2}=|\boldsymbol{z}|^{2}\right\}$.

Let $f_{i}\left(z_{1}, z_{2}\right)(i=1,2)$ be non-degenerate weighted homogeneous polynomials with weights $\left(r_{i 1}, r_{i 2}\right), 0<r_{i 1} \leqq r_{i 2} \leqq 1 / 2$, such that $\left(C^{2}, f_{1}^{-1}(0)\right.$ is relatively homeomorphic to $\left(\boldsymbol{C}^{2}, f_{2}^{-1}(0)\right)$. By King [1] we may assume that there exists a homeomorphism $h: \varepsilon S^{3} \rightarrow \varepsilon S^{3}$ such that $h\left(f_{1}^{-1}(0) \cap \varepsilon S^{3}\right)=f_{2}^{-1}(0) \cap \varepsilon S^{3}$ for sufficiently small number $\varepsilon>0$. Since $0<r_{i 1} \leqq r_{i 2} \leqq 1 / 2$, the weights are invariant under coordinate transformations (Saito [6]). So we can assume that there exists at least a point $z_{i}=\left(z_{i 1}, z_{i 2}\right) \in f_{1}^{-1}(0) \cap \varepsilon S^{3}$ such that $z_{i 1} \cdot z_{i 2} \neq 0$ for $i=1,2$. Then we have

ASSERTION 3. $h\left(\boldsymbol{C}_{f_{1}}^{*}\left(\boldsymbol{z}_{1}\right)\right)=\boldsymbol{C}_{f_{2}}^{*}\left(h\left(\boldsymbol{z}_{1}\right)\right)$.

Proof of Assertion 3. Each connected component $K_{l}^{i}$ of $f_{\imath}^{-1}(0) \cap \varepsilon S^{3}$ is the set $\boldsymbol{C}_{f_{i}}^{*}(\boldsymbol{z})$, where $\boldsymbol{z}$ is any point of $K_{l}^{i}$. For each component $K_{l}^{1}$ of $f_{1}^{-1}(0) \cap \varepsilon S^{3}$, $h\left(K_{l}^{1}\right)$ is a component of $f_{2}^{-1}(0) \cap \varepsilon S^{3}$. Thus the assertion follows.

On the other hand, by the topological invariance of characteristic polynomials ([2]) and the explicit form of characteristic polynomials associated with nondegenerate weighted homogeneous polynomials $([3])$, we have $\left[b_{11}, b_{12}\right]=\left[b_{21}, b_{22}\right]$.

Now we consider two cases to complete the proof of the theorem.

Case I. $a_{11} \cdot\left[b_{11}, b_{12}\right] / b_{11} \neq 1$.

By Assertions 2, 3 and Schreier's theorem (see [5] p. 54),

$$
\begin{aligned}
& a_{11} \cdot\left[b_{11}, b_{12}\right] / b_{11}=a_{21} \cdot\left[b_{21}, b_{22}\right] / b_{21}, \\
& a_{12} \cdot\left[b_{11}, b_{12}\right] / b_{12}=a_{22} \cdot\left[b_{21}, b_{22}\right] / b_{22} .
\end{aligned}
$$

From these equalities and $\left[b_{11}, b_{12}\right]=\left[b_{21}, b_{22}\right]$, we have

$$
a_{11} / b_{11}=a_{21} / b_{21} \text { and } a_{12} / b_{12}=a_{22} / b_{22} .
$$

Case II. $a_{11} \cdot\left[b_{11}, b_{12}\right] / b_{11}=1$.

In this case we have $a_{11}=a_{21}=1, b_{11}=\left[b_{11}, b_{12}\right]=\left[b_{21}, b_{22}\right]=b_{21}$. Since Milnor number, which is topologically invariant ([2]), is $\left(b_{1} / a_{1}-1\right) \cdot\left(b_{2} / a_{2}-1\right)$ for a non-degenerate weighted homogeneous polynomial with weights $\left(a_{1} / b_{1}, a_{2} / b_{2}\right)$ ([3]), we have

$$
a_{12} / b_{12}=a_{22} / b_{22} \text {. }
$$

These cases complete the proof.

\section{REFERENCES}

[1] H. KING, Topological type of isolated critical points, Ann. of Math., 107 (1978), 385-397. 
[2] LÊ DŨNG TRÁNG, Topologie des singularities des hypersurfaces complexes, Asterisque, 7 et 8 (1972), 171-182.

[3] J. MILNOR AND P. ORLIK, Isolated singularities defined by weighted homogeneous polynomials, Topology, 9 (1970), 385-393.

[4] P. ORLIK, Weighted homogeneous polynomials and fundamental groups, Topology, 9 (1970), 267-273.

[5] D. Rolfsen, Knots and Links, Publish or Perish, (1976).

[6] K. SAIto, Quasihomogene isolierte Singularitaten von Hyperflachen, Invent. Math., 14 (1971), 123-142.

[7] E. Yoshinaga AND M. Suzuki, Topological types of quasihomogeneous singularities in $C^{2}$, Topology, 18 (1979), 113-116.

Department of Mathematics

SCHOOL OF SCIENCE

AND ENGINEERING

WASEDA UNIVERSITY

SHINJUKU, TOKYo

JAPAN 